\title{
Acerca de unas presuntas novedades taxonómicas y corológicas de gramíneas para Extremadura (España)
}

\section{Carlos Romero-Zarco}

Departamento de Biología Vegetal y Ecología, Universidad de Sevilla, España.

\section{Correspondencia}

e-mail: zarco@us.es

Recibido: 12 febrero 2021

Aceptado: 22 febrero 2021

Publicado on-line: 26 febrero 2021

Editado por: Marta Recio Criado

\section{Resumen}

Se discuten ciertos datos taxonómicos y corológicos de nueve taxones de gramíneas (Poaceae) de los géneros Anthoxanthum, Arrhenatherum, Avena y Helictochloa publicados recientemente en relación con la flora de Extremadura (España).

Palabras clave: Gramíneas, Poaceae, taxonomía, distribución, Extremadura, España.

\begin{abstract}
About some alleged taxonomic and chorological novelties of grasses for Extremadura (Spain).

Certain taxonomic and chorological data about nine taxa of grasses (Poaceae) of the genera Anthoxanthum, Arrhenatherum, Avena and Helictochloa recently published in relation to the flora of Extremadura (Spain) are discussed.
\end{abstract}

Key words: Grasses, Poaceae, taxonomy, chorology, Extremadura, Spain.
Extremadura es una de las regiones peninsulares mejor estudiadas en relación con la flora agrostológica. Esta posición privilegiada se debe en gran parte a los resultados de los proyectos y estudios dirigidos por el Prof. J.A. Devesa en la Universidad de Extremadura entre 1986 y 2004, siendo de destacar la monografía sobre las gramíneas extremeñas (Devesa, 1991). Con la intención de aportar nuevos datos a este acervo regional, se publicó en 2019 un artículo (Vázquez et al., 2019) con novedades que sorprendieron a algunos de los que estábamos por entonces editando gramíneas para Flora iberica. En esta y en una publicación anterior (Vázquez \& Cabeza, 2009), se dan a conocer novedades sobre gramíneas de Extremadura, algunas de las cuales han resultado ser errores de identificación. Tal es el caso de Poa guadianensis (F.M. Vázquez) F.M. Vázquez in Folia Bot. Extremadurensis 13: 89 (2019) [Poa flaccidula subsp. guadianensis F.M. Vázquez in Folia Bot. Extremadurensis 9: 66 (2016)], que según Ortega Olivencia (2020) se basa en un ejemplar inmaduro posiblemente de $P$. bulbosa; o el caso de Glyceria striata (Lam.) Hitchc., que según López et al. (2018) resultó ser la muy común Poa infirma Kunth; o el caso de Bromus parvispiculatus $\mathrm{H}$. Scholz, que según Acedo \&
Llamas (en prensa) parece corresponder a $B$. intermedius Guss., especie muy rara en la Península que ya había sido citada en Badajoz (Vázquez \& Scholz, 2008).

En consecuencia, era necesario comprobar las novedades más destacadas por si aún había tiempo de añadirlas al volumen de gramíneas de Flora iberica en la fase de corrección de pruebas. Para ello se solicitaron en préstamo los materiales necesarios, que fueron amablemente enviados por el personal del Herbario del Centro La Orden (Badajoz, HSS). De los 8 pliegos recibidos ninguno resultó estar correctamente identificado, tal como se expone a continuación.

\section{Anthoxanthum amarum Brot.}

Especie endémica del noroeste de España y norte de Portugal. Se han examinado dos de los tres testimonios publicados como novedad para la provincia de Cáceres. Uno procedente de Hervás (HSS 54554) y otro de La Garganta a Baños de Montemayor (HSS 23327). Ambos se identifican sin dificultad con Anthoxanthum odoratum L., especie yaconocida en la región, que se diferencia de $A$. amarum por sus hojas relativamente estrechas y por la ausencia de estolones o pequeños tubérculos basales (Lema et al., 2019). 
Arrhenatherum elatius subsp. baeticum RomeroZarco

Esta subespecie se distribuye principalmente por el este de la Península (Sistemas Béticos e Ibérico) y Baleares (Ibiza), con escasas localizaciones en enclaves calcáreos del norte de España y centro-oeste de Portugal (RomeroZarco, 2019). El material examinado: Badajoz, Castuera, 30-IV-2019, F. Márquez \& F.M. Vázquez (HSS 75067), se identifica claramentecon A. album var. album, taxón común en la zona.

Avena barbata subsp. hirtula (Lag.) Tab. Morais

En España, este taxón se distribuye exclusivamente por el SE árido (Romero-Zarco, 1990). Los dos pliegos citados de la provincia de Badajoz (HSS 63727 y HSS 64388) corresponden a la más común subespecie barbata. Otro pliego citado de Cáceres no se ha podido estudiar pero no merece credibilidad a juzgar por el sitio donde se recolectó ("margen de charcas").

\section{Avena longiglumis Durieu}

Esta especie es característica de arenales del litoral, aunque se conocen algunas poblaciones en el interior, pero solo en los alrededores del valle del Guadalquivir (Romero-Zarco, 1996). La cita para Badajoz (HSS 64054) corresponde a una planta con espiguillas inmaduras de Avena sterilis L. subsp. sterilis.

Helictochloa albinervis (Boiss.) Romero Zarco

En el artículo que aquí se comenta, aparece esta especie como novedad regional, aunque sin añadir material alguno. Para Extremadura solo hemos visto en los herbarios españoles plantas de la subsp. gaditana (Romero Zarco) Romero Zarco in Acta Bot. Malacitana 39: 308 (2014). La subsp. albinervis solo se ha confirmado para las provincias españolas de Cádiz y Málaga y en puntos concretos de algunas regiones del oeste de Portugal (RomeroZarco, 2020).

Helictochloa delicatula (Franco) F.M. Vázquez in Folia Botanica Extremadurensis 13(2): 78 (2019) Avenula delicatula Franco in Bot. J. Linn. Soc. 76: 359(1978)

Se trata de una forma enana y cacuminal del complejo de H. marginata (Love) Romero Zarco, descrita de los suelos peridotíticos del $\mathrm{N}$ de Portugal, que ha sido descrita como variedad para el Sistema Central [Avenula sulcata var. reuteri Romero Zarco in Lagascalia 14: 122 (1984)] y como subespecie en los Pirineos [Avenula marginata subsp. pyrenaica Holub in Preslia 49(3): 208 (1977)]. La combinación nomenclatural es legítima, pero de escasa utilidad. Su nombre correcto en el rango varietal es Helictochloa marginata var. reuteri (Romero Zarco) Romero Zarco in Acta Bot. Malacitana 40: 294 (2015).

Helictochloa discreta F.M. Vázquez in Folia Botanica Extremadurensis 13(2): 79 (2019)

Holotipo: Hs: Cáceres (Cc): Losar de la Vera, cerca del Refugio, subida hacia la Covacha, 30TTK7948, 1371-1382 m.s.n.m., brezal quemado y afloramientos rocosos, 28-IV-2015, D. García \& F.M. Vázquez (HSS64300).

El holotipo es una plantita que se identifica sin duda alguna con Helictochloa marginata var. reuteri, muy frecuente en el Sistema Central (Romero-Zarco, 2015), la misma ya descrita por Amaral Franco en Portugal (véase el párrafo anterior).

Helictochloa pauneroae (Romero Zarco) F.M. Vázquez in Folia Botanica Extremadurensis 13(2): 78(2019) ["pauneroi"]

H. bromoides subsp. pauneroae (Romero Zarco) Romero Zarco.

Los escasos argumentos que alega el autor para elevar al rango específico este taxón no se ajustan a la realidad, ya que se basan en datos obsoletos. La separación de la subespecie pauneroae, que en su origen se estableció en la ausencia de pubescencia en el lema, fue posteriormente modificada por M. Röser (1989) en el sentido de admitir para este taxón solo plantas estoloníferas de zonas montañosas del SE peninsular, criterio seguido en Flora iberica XIX (Romero-Zarco, 2018; 2020: 520). Posteriormente, han aparecido ejemplares estoloníferos en otras zonas; algunos de dichos ejemplares se han identificado con ciertas dudas, debido a que su recolección es incompleta. Tal es el caso de un único ejemplar de Badajoz: Entre Zafra y Llerena, km 94 (MA 453461). En el artículo que aquí se comenta no se indican materiales de la especie, que es muy rara en Extremadura. Los que se han estudiado son de plantas cespitosas con lemas glabros que identificamos con la subespecie bromoides [Badajoz: Alconera, pastizales higrocalizos (UNEX 11612); Cerro de Los Santos de Maimona (MA 453462); Llerena (UNEX 11611)]. Hay una cita anterior para el norte de la provincia de Cáceres (Vázquez et al., 2004) que no se ha podido confirmar, pero a juzgar por las que se han visto del sur de Salamanca debe corresponder también a la subespecie típica.

Helictochloa occidentalis (Gervais) F.M. Vázquez in Folia Botanica Extremadurensis 13(2): 78 (2019), nom. illeg.

La invalidez del basiónimo fue explicada hace décadas por M. Röser (1992). La forma de trabajar de C. Gervais, por otra parte un excelente investigador y pionero de la taxonomía del género, no incluía la indicación de material de herbario 
como tipo nomenclatural, sino que hacía referencia a plantas cultivadas que no se conservaron, procedimiento que infringe el art. 8.4 del Código (Greuter \& Rankin Rodríguez, 2018). El nombre correcto para este taxón en la categoría de especie es Helictochloa cintrana (Röser) Romero Zarco.

Además, la diagnosis que justificaría la correcta identificación del taxón (Vázquez et al. loc. cit.: 80 ) hace referencia a un carácter trivial y muy variable, que no permite diferenciar esta especie de otras próximas. Por tanto, los materiales extremeños citados bajo tal nombre podrían corresponder con más probabilidad a $\mathrm{H}$. albinervis subsp. gaditana, cuya morfología es muy similar (Romero-Zarco, 2014).

\section{Agradecimientos}

Agradezco al Herbario del Centro La Orden (Badajoz, HSS) el envío en préstamo de los materiales estudiados en estas notas.

\section{Bibliografía}

Acedo, C. \& Llamas, F. (en prensa). Bromus L. En C.Romero Zarco et al. (eds.), Flora iberica vol. XIX(II) Gramineae (partim). Real Jardín Botánico,CSIC, Madrid.

Devesa, J.A. (Ed., 1991). Las Gramíneas de Extremadura. Badajoz: Servicio de Publicaciones de la Universidad de Extremadura.

Greuter, W. \& Rankin Rodríguez, R. (ds.) (2018). Código Internacional de Nomenclatura para algas, hongos y plantas (Código de Shenzhen), 19ㅇ Congreso Internacional de Botánica Shenzhen, China (2017). Edición en español. Berlín: Fundación Herbarium Greuter.

Lema, I, Pimentel, M. \& Sahuquillo, E. (2019). Anthoxanthum L., borrador para Flora iberica no 8. En C. Romero-Zarco (actualizado periódicamente), Gramina Iberica.

https://sites.google.com/site/graminaiberica. Consultado el 2-feb-2021.

López, J., Ortega-Olivencia, A., Rodríguez-Riaño, T. \& Devesa, J.A. (2018). Notas florísticas sobre algunas gramíneas de Flora iberica. Acta Botanica Malacitana, 43, 171-174.

Ortega Olivencia, A. (2020). Poa L. En J.A. Devesa etal. (Eds.), Flora iberica vol. XIX(I) Gramineae (partim): 113-152. Real Jardín Botánico, CSIC, Madrid.

Romero-Zarco, C. (1990). Las avenas del grupo barbata en la Península Ibérica y Baleares. Lagascalia, 16(2), 243-268.
Romero-Zarco, C. (1996). Sinópsis del género Avena L. (Poaceae, Aveneae) en España peninsular y Baleares. Lagascalia, 18(2), 171-198.

Romero-Zarco, C. (2014). Notas taxonómicas sobre el género Helictochloa Romero Zarco (Poaceae). Acta Botanica Malacitana, 39, 308-310.

Romero-Zarco, C. (2015). Variedades enanas del complejo Helictochloa albinervis- $H$. marginata (Poaceae). Acta Botanica Malacitana, 40, 292295.

Romero-Zarco, C. (2018). Helictochloa Romero Zarco, borrador no 12 para Flora iberica. En C. Romero-Zarco (actualizado periódicamente), Gramina Iberica.

https://sites.google.com/site/graminaiberica Consultado el 2-feb-2021.

Romero-Zarco, C. (2019). Arrhenatherum P.B. Beauv., borrador no 8 para Flora iberica. En C. Romero- Zarco (actualizado periódicamente), Graminalberica.

https://sites.google.com/site/graminaiberica Consultado el 2-feb-2021.

Romero-Zarco, C. (2020). Helictochloa Romero Zarco. En J.A. Devesa et al. (Eds.), Flora iberica vol. XIX(I) Gramineae (partim): 515-546. Real Jardín Botánico, CSIC, Madrid.

Röser, M. (1989) Karyologische, systematische und chorologische Untersuchungen an der Gattum Helictotrichon Besser ex Schultes \& Schultes im westlichen Mittelmeergebiet. Dissertationes Botanicae, 145, 1-250.

Röser, M. (1992). Helictotrichon cintranum species nova, a rare south-west European oat grass (Poaceae: Pooideae: Aveneae). Taxon, 41, 6061.

Vázquez, F.M. \& Cabeza, M. (2009). Anotaciones corológicas y taxonómicas a la flora en Extremadura. 23. Glyceria striata (Lam.) A.S. Hitch. Folia Botanica Extremadurensis, 4, 64-65.

Vázquez, F.M. \& Scholz, H. (2008). Anotaciones al género Bromus L. subgen. Bromus (Poaceae) en Extremadura (España), Folia Botanica Extremadurensis, 2, 11-30.

Vázquez, F.M., García Alonso, D., Márquez García, F. \& Guerra Barrena, M.J. (2019). Anotaciones a la diversidad de la familia Poaceae (Gramineae) en Extremadura (España). Folia Botanica Extremadurensis, 13(2), 39-130.

Vázquez, F.M., Ramos, S., Lucas, A. B., García, D. \&Blanco, J. (2004). Fragmentos taxonómicos, corológicos, nomenclaturales y fitocenológicos (135-145). 138. Aportaciones a la flora de Extremadura (España). Acta Botanica Malacitana, 29, 265-268. 